\title{
SIMULAÇÕES NUMÉRICAS EM ALTA RESOLUÇÃO DE UM COMPLEXO CONVECTIVO DE MESOESCALA COM DIFERENTES CONDIÇÕES INICIAIS E DE CONTORNO
}

\author{
Daniel Caetano Santos ${ }^{1 *}$, Ernani de Lima Nascimento ${ }^{1}$ \\ ${ }^{1}$ Departamento de Física, Universidade Federal de Santa Maria \\ *danielcae@gmail.com
}

\section{RESUMO}

O objetivo deste trabalho é o de avaliar o desempenho do modelo WRF na simulação da precipitação causada por um CCM ocorrido entre os dias 18 e 19 de Janeiro de 2010 sobre a Bacia do Prata, com acumulado diário observado superior a $100 \mathrm{~mm}$. Para as simulações foram utilizadas duas diferentes bases de dados do CFSR (reanálise) e GFS (análise) para as condições iniciais e de contorno (CI-CC). As simulações utilizando o CI-CC/CFSR, apresentaram melhor desempenho na simulação da precipitação e na descrição do ciclo de vida do sistema.

\section{SUMMARY}

The goal of this work is to evaluate the performance of the WRF model on the rainfall simulation associated with a MCC that occurred between 18 and 19 December 2010 affecting the La Plata Basin, with observed rainfall accumulation above $100 \mathrm{~mm}$ in $24 \mathrm{hrs}$. Two different data bases were used to provide ICs and BCs to the simulations: the CFS Reanalysis (CFSR) and analysis from the GFS model. Simulations using ICs-BCs provided by the CFSR displayed better performance in describing the rainfall accumulation and the system's life cycle.

\section{INTRODUÇÃO}

Ocasionalmente sobre o Sudeste da América do Sul (SAS), são observados os Complexos Convectivos de Mesoescala (CCMs; Zipser et al., 2006; Velasco e Fritsch, 1987), importantes para o regime de chuvas do SAS, principalmente na estação quente do ano 
(Durkee et al.,2009). Este aspecto ressalta a importância da correta simulação e previsão numérica (PN) destes eventos na Bacia do Prata.

É possível hoje vislumbrar a PN em uma escala que permite a representação quase explícita da convecção profunda (p.ex., Mittermaier 2013). É desejável avaliar como tais simulações se comportam em função de diversos aspectos computacionais, como a especificação de condições iniciais (CI) e de contorno (CC) (Termonia, 2003; Errico et al., 1993).

Este estudo avalia simulações de alta resolução (i.e., com convecção quase explícita) com o modelo numérico Weather Research \& Forecasting (WRF) para um caso de CCM sobre o SAS, utilizando duas diferentes fontes de CI-CC.

\section{DADOS E MÉTODOS}

Foram realizadas duas simulações de um caso de CCM com o modelo WRF v3.5, com cada simulação contendo o aninhamento de grades em domínios de 48 (G1), 12 (G2) e 4 (G3) km de espaçamento horizontal de grade. As principais configurações do modelo são mostradas na Tabela 1. A grade G3 é a de convecção quase explícita e todas as três grades têm 29 níveis verticais. Cada simulação na grade G1 partiu de uma fonte distinta de CI-CC, que são: a) Climate Forecast System Reanalysis (CFSR, Saha et al., 2010) e; b) Global Forecast System Analysis (GFSanl). A descrição das condições de contorno encontra-se na Tabela 2.

O CCM estudado ocorreu entre os dias 18 e 19 de Janeiro de 2010, atingindo a Argentina, o Uruguai, o Paraguai e o sul do Brasil, gerando acumulados superiores a 100mm em 24 horas, conforme os dados MERGE (Rozante et al. 2010; também empregado como referência para avaliação das simulações). É avaliada a precipitação resultante das simulações na grade de $4 \mathrm{~km}$ enfatizando a sensibilidade à fonte de CI-CC empregada.

Tabela 1 - Principais parametrizações físicas utilizadas no WRF

\begin{tabular}{|c|c|c|c|c|c|}
\hline \multicolumn{6}{|c|}{ Parametrizações } \\
\hline \multirow{2}{*}{ Girade } & \multirow{2}{*}{ Convecça } & \multirow{2}{*}{ Camada Limite } & \multicolumn{2}{|c|}{ Radilaçăo } & \multirow{2}{*}{ Microfísical } \\
\hline & & & Ondala Longa & Ondala Curta & \\
\hline $48 \mathrm{Km}$ & Kain-Fritsch & Hong et al. (2006) & RRTM & Dudhia & Lin et al. (1983) \\
\hline $12 \mathrm{Km}$ & Kain-Fritsch & Hong et al. (2006) & RRTM & Dudhia & Lin et al. (1983) \\
\hline $4 \mathrm{Km}$ & - & Hong et al. (2006) & RRTM & Dudhia & Lin et al. (1983) \\
\hline
\end{tabular}


Tabela 2 - Descrição dos dados de CI-CC para a grade G1

\begin{tabular}{|c|c|c|c|}
\hline \multicolumn{4}{|c|}{ Condições de Contorno } \\
\hline \multirow{2}{*}{ Fonte } & \multicolumn{2}{|c|}{ Resoluçẵo } & \multirow{2}{*}{ Niveis Verticais } \\
\cline { 2 - 3 } & Horizontal & Temporal & $\mathbf{3 7}$ \\
\hline CFSR & $\mathbf{0 . 5}^{\bullet}$ & $\mathbf{6 h}$ & $\mathbf{2 6}$ \\
\hline GFSanl & $\mathbf{1}^{\bullet}$ & $\mathbf{6 h}$ & $\mathbf{6 h}$ \\
\hline
\end{tabular}

\section{RESULTADOS}

Os resultados mostram que o total pluviométrico acumulado em $24 \mathrm{~h}$ simulado pelo WRF/CFSR apresentou os valores mais próximos aos das observações para o dia 19 de janeiro, principalmente sobre o oeste do Rio Grande do Sul e do centro ao nordeste da Argentina (Figura 2D-F).

$\mathrm{Na}$ análise do acumulado horário (00Z, 06Z, 12Z e 18Z) em comparação com as respectivas imagens do satélite GOES no canal IR-realçado (figuras não mostradas), é possível observar que o WRF/CFSR registrou chuva no local e hora de início do desenvolvimento do CCM (18 de Janeiro de 2010 às 12Z), o que não foi registrado pelo WRF/GFS, mostrando a diferença na simulação do desenvolvimento do sistema.

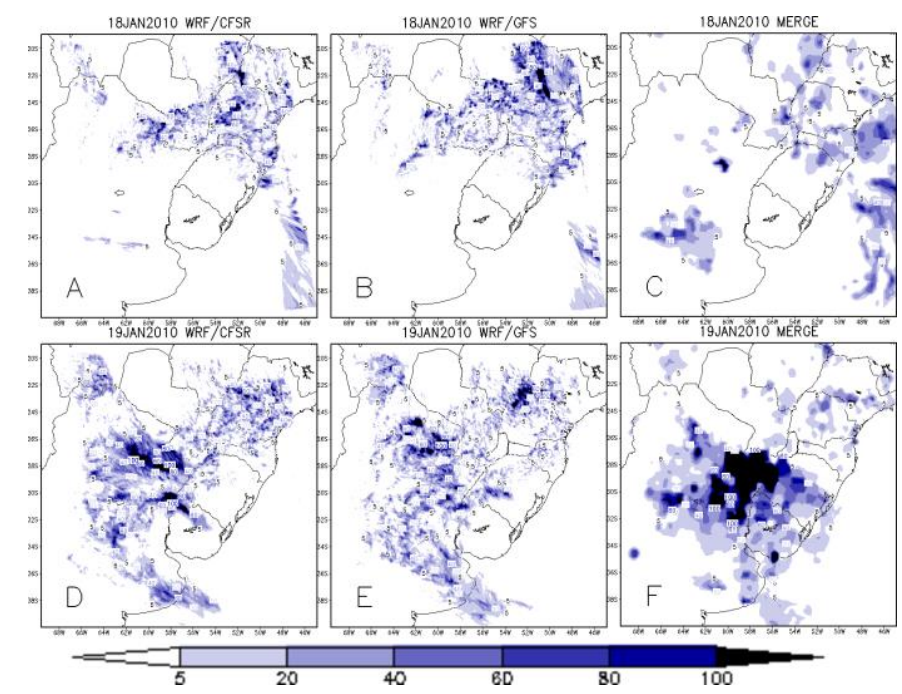

Figura 1 - Total acumulado em 24h simulados pelo WRF/CFSR (A,D), WRF/GFSanl (B,E) e Observados MERGE (C,F), para os dias 18 e 19 de Janeiro de 2010, respectivamente.

\section{CONCLUSÕES}

Os resultados sugerem que as simulações utilizando CI-CC de maior resolução horizontal e 
com um maior número de níveis verticais (CFSR) apresentaram um melhor desempenho em simular a precipitação causada pelo CCM de estudo, além de uma melhor descrição do ciclo de vida do sistema.

Este resultado indica que a maior resolução espacial dos dados de CI-CC pode ter sido um fator predominante na simulação do sistema fazendo com que o modelo de mesoescala acompanhasse com melhor destreza o CCM.

\section{AGRADECIMENTOS}

Os autores agradecem ao Projeto SIMTECO (FINEP) pela disponibilização do Cluster para as simulações utilizadas neste estudo.

\section{REFERÊNCIAS}

DURKEE, J. D.; MOTE, T. L.; SHEPHERD, J. M. The contribution of mesoscale convective complexes to rainfall across subtropical South America. Journal of Climate, v. 22, n. 17, p. 4590-4605, 2009.

ERRICO, R. M.; VUKICEVIC, T.; RAEDER K. Comparison of initial and lateral boundary condition sensitivity for a limited-area model. Tellus, v.45A, n.5, p.539-557, 1993.

MITTERMAIER, M. P. A strategy for verifying near-convection-resolving model forecasts at observing sites. Monthly Weather Review, DOI: 10.1175/WAF-D-12-00075.1, 2013. No prelo.

TERMONIA, P. Monitoring and improving the temporal interpolation of lateral boundary coupling data for limited area models. Monthly Weather Review, 131, 2450-2463, 2003. ROZANTE, J.; MOREIRA, D.; GONÇALVES, L. G.; VILA, D. Combining TRMM and surface observations of precipitation: Technique and validation over South America.

Weather and Forecasting, v. 25, n. 3, p. 885-894, 2010.

VELASCO, E.; FRITSCH, J. M. Mesoscale convective complexes in the Americas. Journal of Geophysical Research, v. 92, n. D8, p. 9591-9613, 1987.

ZIPSER, E.; CECIL, D.; LIU, C.; NESBITT, S.; YORTY, D. Where are the most intense thunderstorms on Earth? Bull. Amer. Meteor. Soc., v. 87, p. 1057-1071, 2006. 